\title{
Asymmetric Raman lines caused by an anharmonic lattice potential in lithium niobate
}

\author{
U. T. Schwarz and Max Maier \\ NWF II-Physik, Universität Regensburg, D-93040 Regensburg, Germany
}

(Received 20 September 1996)

\begin{abstract}
Spontaneous Raman scattering experiments were performed in stoichiometric and congruent $\mathrm{LiNbO}_{3}$, $\mathrm{MgO}: \mathrm{LiNbO}_{3}$, and $\mathrm{ZnO}: \mathrm{LiNbO}_{3}$ in the temperature range from 20 to $500 \mathrm{~K}$. The temperature dependent broadening and asymmetry of the Raman lines of the optical phonons with $A_{1}$ symmetry is interpreted as the superposition of a series of Raman lines caused by transitions between higher levels in the anharmonic potential for the ionic motion and the decay of the optical phonon into two acoustic phonons. The Raman linewidths in the various doped and undoped $\mathrm{LiNbO}_{3}$ crystals differ by a temperature independent scattering term. [S0163-1829(97)09017-6]
\end{abstract}

Lithium niobate has important technical applications, but is equally well the subject of fundamental studies in nonlinear optics and electro-optics. It is also a model substance for the ferroelectric phase transition. The four optical phonons with $A_{1}$ symmetry in $\mathrm{LiNbO}_{3}$ at $251,273,331$, and $631 \mathrm{~cm}^{-1}$ correspond to a movement of the ions parallel to the optical axis of the crystal and are thus connected with the ferroelectric phase transition. Spontaneous Raman scattering experiments of the $A_{1}$ modes were the major tools in the search for a soft mode as an evidence for a displacive phase transition, ${ }^{1-3}$ but also for other topics like a microscopic description of the $A_{1}$ modes $^{4}$ or the determination of the Grüneisen constant. ${ }^{5}$ Recent theoretical studies ${ }^{6,7}$ discuss an order/disorder phase transition or some intermediate model for $\mathrm{LiNbO}_{3}$ and the related ferroelectric crystals. These models differ from the potential energy surface for the ionic motion, which should exhibit a single well for the displacive and a double or multiple well for the order/disorder phase transition. ${ }^{6}$ In both cases the potential is asymmetric below $T_{C}$, causing the ferroelectric effect, and has consequently a strong anharmonic part.

Transitions between higher energy levels of the anharmonic potential for the $A_{1}$ (TO) optical phonon, corresponding to the ionic displacement in a unit cell ${ }^{8}$ (Fig. 1), will lead to a series of closely spaced Raman lines beside the original Raman line of the transition $v=0 \rightarrow 1$. This phenomenon causes an asymmetric broadening of the Raman lines, which was first observed in $\mathrm{PbTiO}_{3} .{ }^{9}$ The quantitative analysis of the Raman line shapes provides a measure of the anharmonicity of the potential, which is interesting for calculations of the properties of ferroelectric crystals. There is a growing number of calculations of the energetics of the ferroelectric instability $^{7}$ and on the dielectric response of ferroelectrics ${ }^{6}$ which are based on the anharmonic potentials of the crystals.

In this paper we report on measurements of the dependence of the spontaneous Raman lines of the $A_{1}$ optical phonon modes in $\mathrm{LiNbO}_{3}$ on temperature and composition, with particular emphasis on the line shapes. To analyze the experimental data, the model of the Raman side lines ${ }^{9}$ was extended to include the decay of the optical phonon into two acoustic phonons and the scattering of the optical phonon at crystal imperfections. New results are obtained in the interpretation of the Raman line shapes of undoped congruent
$\mathrm{LiNbO}_{3}$ and $\mathrm{MgO}$ and $\mathrm{ZnO}$ doped $\mathrm{LiNbO}_{3}$. The larger overall Raman linewidths of these crystals compared to stoichiometric $\mathrm{LiNbO}_{3}$ are explained by a temperature-independent term caused by the scattering of the phonons from imperfections, which is different in the different crystals. In contrast, the contribution of the anharmonic decay of the phonons to the Raman linewidth and the parameters of the anharmonic potential, which leads to the side lines, were found to be the same in all investigated crystals.

The spontaneous Raman scattering experiments from 20 to $500 \mathrm{~K}$ were performed with an $\mathrm{Ar}^{+}$laser at $488 \mathrm{~nm}$, a double monochromator (Spex 1402) with a resolution of 3 $\mathrm{cm}^{-1}$, and a photon counting system. Scattering geometry was backward scattering with the polarization of pump and Stokes light parallel to the optical $\hat{c}$ axis of the $\mathrm{LiNbO}_{3}$ crystal, i.e., $x(z z) \bar{x}$ in common notation.

We performed first Raman scattering experiments to con-

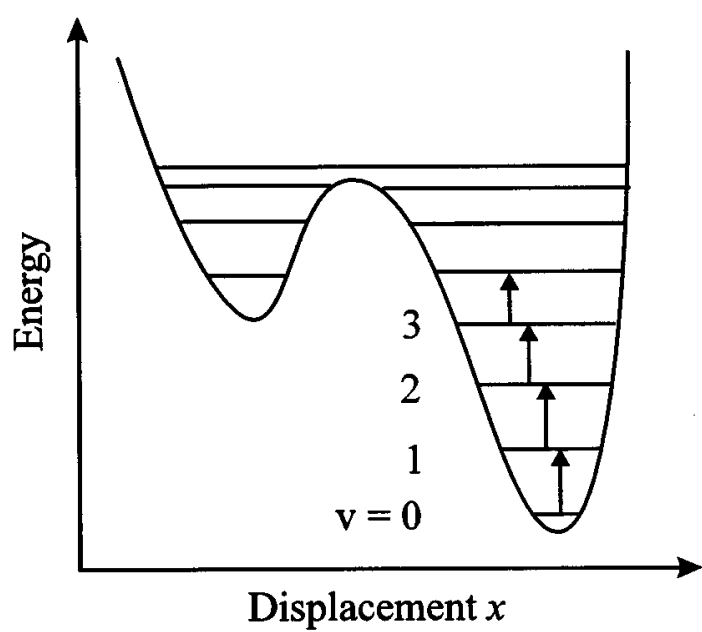

FIG. 1. Schematic representation of the asymmetric double-well potential for the displacement of the ions along the $\hat{c}$ axis in $\mathrm{LiNbO}_{3}$. Because of the anharmonicity, the distance between adjacent energy levels decreases with increasing quantum number $v$, leading to a series of closely spaced Raman lines. The double-well potential is valid for the order/disorder model. In case of a displacive transition it will reduce to a single asymmetric well. 


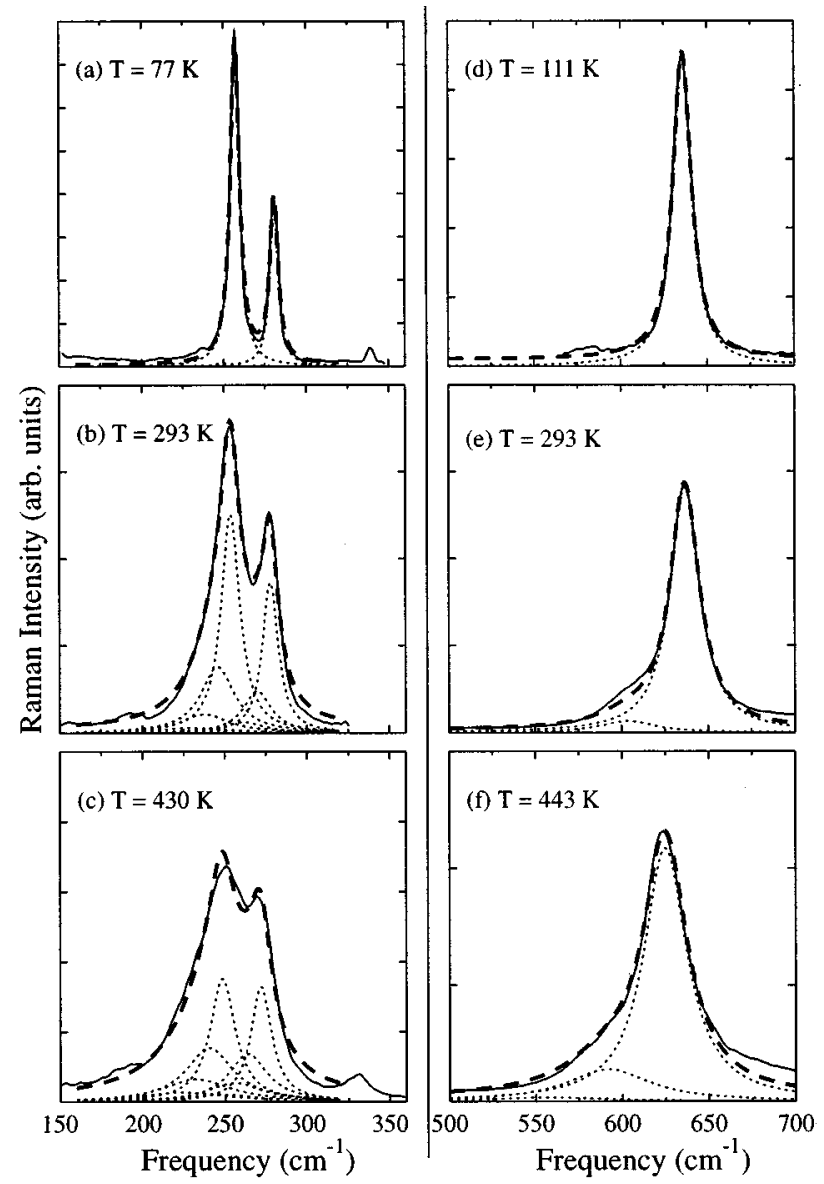

FIG. 2. Spontaneous Raman spectra of the optical phonons with $A_{1}$ symmetry in stoichiometric $\mathrm{LiNbO}_{3}$. The solid lines represent the measured spectra. Three series of calculated Raman lines (dotted lines), caused by the anharmonic potential, contribute to the fit (dashed line) of the Raman spectrum. (a)-(c): 251, 273, and 331 $\mathrm{cm}^{-1}$ modes; (d)-(f): $631 \mathrm{~cm}^{-1}$ mode.

firm the model in stoichiometric $\mathrm{LiNbO}_{3}$, which is particularly suited because of the small Raman linewidths compared to congruent $\mathrm{LiNbO}_{3}$ or doped crystals. Only recently a method was developed to grow large $\left(>5 \times 5 \times 5 \mathrm{~mm}^{3}\right)$ stoichiometric crystals, ${ }^{10}$ which are crystals with a ratio $\mathrm{Li}: \mathrm{Nb}$ of nearly ideal 50:50, with good optical quality from a melt containing potassium.

The solid lines in Figs. 2(a)-2(c) show the measured spontaneous Raman spectra of the 251,273 , and $331 \mathrm{~cm}^{-1}$ $A_{1}$ modes at three different temperatures, picked out of a series of measurements at ten temperature steps between 20 and $500 \mathrm{~K}$. The mode at $331 \mathrm{~cm}^{-1}$ is too weak for a detailed investigation. It will not be discussed further. Linewidths and overlapping of the modes at $251 \mathrm{~cm}^{-1}$ and $273 \mathrm{~cm}^{-1}$ increase with temperature. At temperatures below $100 \mathrm{~K}$ the Raman lines are narrow and symmetric [see, e.g., Fig. 2(a)]. At higher temperatures, the spectra become broader and asymmetric with a more gentle slope at the low frequency side [Figs. 2(b) and 2(c)].

We discuss first the low-temperature region, where the Raman lines are symmetric and correspond to the $v=0 \rightarrow 1$ transition in the anharmonic potential (Fig. 1). We assume that the mechanism, which determines the linewidth $\Gamma$, is the decay of the optical phonon (frequency $\vec{\nu}$ ) into two acoustic phonons with frequency $\bar{\nu} / 2$ and opposite wavevectors ("Klemens channel"). The same mechanism has been successfully applied to describe the frequency dependence of the decay of polaritons in $\mathrm{LiNbO}_{3}$ measured by stimulated Raman scattering. ${ }^{11}$ In this model the temperature dependence of the spontaneous Raman linewidth is given by

$$
\Gamma(\bar{\nu}, T)=\Gamma_{d}\left[2 n\left(\frac{1}{2} \bar{\nu}, T\right)+1\right],
$$

with the phonon population

$$
n(\bar{\nu}, T)=\left[\exp \left(\frac{h c \vec{\nu}}{k_{B} T}\right)-1\right]^{-1} .
$$

The parameter $\Gamma_{d}$ was determined from the measured lowtemperature linewidths, which were corrected by the spectral resolution of the monochromator. We get $\Gamma_{d}=3.4$ and 4.0 $\mathrm{cm}^{-1}$ for the 251 and $273 \mathrm{~cm}^{-1}$ modes, respectively.

In the same model the temperature dependence of the line position $\bar{\nu}$ is given by

$$
\bar{\nu}(T)=\overline{\nu_{0}}-\Delta\left[2 n\left(\frac{1}{2} \overline{\nu_{0}}, T\right)+1\right] .
$$

The parameters $\overline{\nu_{0}}$ and $\Delta$ have been determined by fitting Eq. (2) to the measured positions of the maxima of the Raman lines from 20 to $500 \mathrm{~K}$. Good fits were obtained for $\bar{\nu}_{0}=261$ $\mathrm{cm}^{-1}, \Delta=2.6 \mathrm{~cm}^{-1}$ and $\overline{\nu_{0}}=285 \mathrm{~cm}^{-1}, \Delta=2.6 \mathrm{~cm}^{-1}$.

At temperatures $T>200 \mathrm{~K}$ the Raman lines are asymmetric, the asymmetry becoming more pronounced at higher temperatures. We discuss the asymmetry in terms of side lines caused by the transitions $v=1 \rightarrow 2,2 \rightarrow 3$, etc. in the anharmonic potential shown in Fig. 1. ${ }^{9}$ The potential is temperature dependent and influenced by the local electric field. ${ }^{6}$ However, since our investigated temperature range (20 to $500 \mathrm{~K}$ ) is well below the Curie temperature of $\mathrm{LiNbO}_{3}$ $\left(T_{C}=1480 \mathrm{~K}\right)$ and only the lowest states of the potential are populated, we neglect these effects. For the description of the experimental results we need the distance $\Delta \bar{\nu}$ between the side lines, their intensities, and linewidths. For simplicity we assume that the anharmonic part of the potential is proportional to $x^{3}$, where $x$ is the normal coordinate. The energy derived by perturbation calculations in second $\operatorname{order}^{12}$ is given by

$$
E(v)=h c \bar{\nu}\left(v+\frac{1}{2}\right)-h c \bar{\nu} x_{e}\left(v+\frac{1}{2}\right)^{2},
$$

yielding a distance of the $v$ th side line (corresponding to the $v \rightarrow v+1$ transition) to the main line $(0 \rightarrow 1$ transition $)$ of

$$
\Delta \bar{\nu}_{v}=v \Delta \bar{\nu}=-2 v \bar{\nu} x_{e} .
$$

In this approximation the frequency distance $\Delta \bar{\nu}$ between the side lines is constant and proportional to the anharmonicity coefficient $x_{e}$. This is the only fit parameter for the side lines.

The intensity ratio $R$ of the $v$ th side line and the main line is determined by the square of the matrix element of the transition, which is proportional to $v+1$ in the harmonic approximation, and by the relative population of the $v$ th level. ${ }^{9}$ It is given by

$$
R=(v+1) \exp \left(-\frac{v h c \vec{\nu}}{k_{B} T}\right) .
$$


In the Boltzmann factor also the harmonic approximation for the potential has been used.

The relaxation times of the higher energy levels in the potential are expected to be shorter, leading to a larger linewidth of the side lines. We assume the linewidth of the $v$ th side line to be given by

$$
\Gamma_{v}=\Gamma(v+1),
$$

where $\Gamma$ is the width of the main line. The corresponding relation for the relaxation rate constants of higher vibrational levels of molecules has been confirmed experimentally in Ref. 13.

We have calculated the shape of the Raman lines at different temperatures using the model for the main and side lines described above. We used the numbers of $\Gamma_{d}, \bar{\nu}_{0}$, and $\Delta$ determined for the main line at lower temperature, took into account three side lines and fitted the value of $x_{e}$ for the side lines to get good agreement between the calculated and measured Raman lines. We used values of $x_{e}=0.016$ and 0.015 for the 251 and $273 \mathrm{~cm}^{-1}$ modes, respectively, which correspond to a distance $\Delta \bar{\nu}=8 \mathrm{~cm}^{-1}$ between the side lines. These values of $x_{e}$ in $\mathrm{LiNbO}_{3}$ are smaller than that for the $148 \mathrm{~cm}^{-1}$ mode in $\mathrm{PbTiO}_{3}$, where the distance between the side lines corresponds to a value $x_{e}$ of about $0.04 .{ }^{9}$

Figures 2(a)-2(c) show typical examples of the Raman lines of the 251 and $273 \mathrm{~cm}^{-1}$ modes at different temperatures. The dotted lines represent the calculated main and side lines. They are added up to give the dashed line, which shows good agreement with the measured spectra (solid line). The asymmetry of the Raman lines is seen to be caused by the side lines. The contribution of phonon scattering at imperfections was found to be negligible for the lowfrequency modes in stoichiometric $\mathrm{LiNbO}_{3}$. At the high end of the temperature range $T>450 \mathrm{~K}$ an additional broadening by scattering at thermal phonons has been observed.

The Raman line of the $631 \mathrm{~cm}^{-1}$ mode is shown at three temperatures in Figs. 2(d)-2(f). The temperature dependence of the main line is again described by the symmetric decay of the optical phonon. However, an additional term corresponding to scattering at a thermal phonon with a frequency $\bar{\nu}_{t}$ (Ref. 14) was added to get good agreement with the experimental results:

$$
\Gamma(\bar{\nu}, T)=\Gamma_{d}\left[2 n\left(\frac{1}{2} \bar{\nu}\right)+1\right]+\Gamma_{s}\left[n\left(\overline{\nu_{t}}\right)-n\left(\overline{\nu_{t}}+\bar{\nu}\right)\right] .
$$

Best agreement for the $\bar{\nu}=631 \mathrm{~cm}^{-1}$ mode was obtained for $\Gamma_{d}=9.3 \mathrm{~cm}^{-1}, \Gamma_{s}=10.4 \mathrm{~cm}^{-1}$, and $\bar{\nu}_{t}=251 \mathrm{~cm}^{-1}$. In this three phonon scattering process, two optical phonons at 631 $\mathrm{cm}^{-1}$ and $251 \mathrm{~cm}^{-1}$ are annihilated and one at $882 \mathrm{~cm}^{-1}$ $\left[A_{1, \mathrm{LO}}\right.$ or $E_{\mathrm{LO}}($ Ref. 15$\left.)\right]$ is created. But probably more scattering and decay processes contribute to the linewidth. Due to the high frequency of the $631 \mathrm{~cm}^{-1}$ mode, only two side lines are important in the examined temperature range. The fitting procedure is the same as described above. We found best agreement between the calculations and the measurements for $x_{e}=0.026$, corresponding to a distance $\Delta \bar{\nu}=33$ $\mathrm{cm}^{-1}$ of the side lines [see Figs. 2(d) $\left.-2(\mathrm{f})\right]$.

We have also measured the Raman lines of the $A_{1}$ modes in undoped congruent $\mathrm{LiNbO}_{3}(\mathrm{Li}: \mathrm{Nb}$ ratio 48.6:51.4) and in $\mathrm{MgO}$ and $\mathrm{ZnO}$ doped $\mathrm{LiNbO}_{3}$. The usual way ${ }^{1,4}$ to determine the linewidths of the 251 and $273 \mathrm{~cm}^{-1}$ modes is a decom-

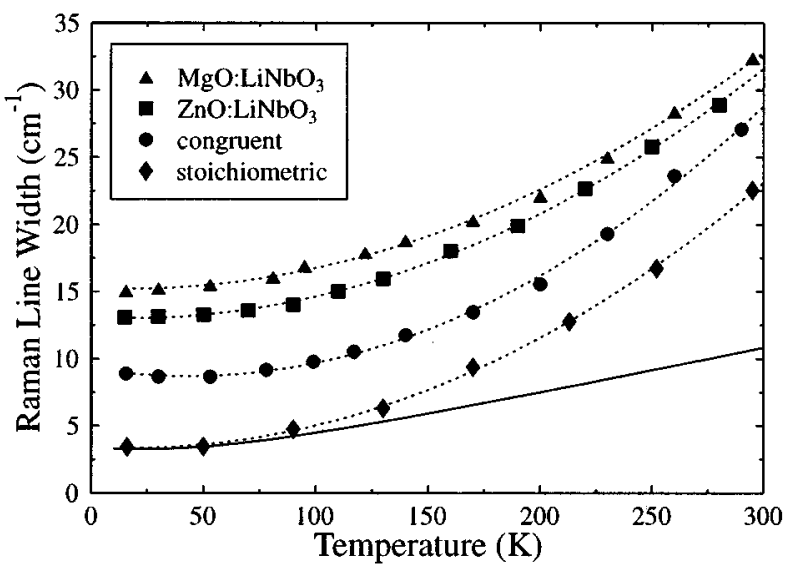

FIG. 3. Temperature dependence of the linewidth of the 251 $\mathrm{cm}^{-1} A_{1}$ phonon for different $\mathrm{LiNbO}_{3}$ crystals. The solid line has been calculated from the decay of the optical phonon into two acoustic phonons [Eq. (1)]. The dotted lines are guide lines for the eye.

position into two Lorentzian lines, neglecting the asymmetry. The width (full width at half maximum) of the corresponding Lorentzian line of the $251 \mathrm{~cm}^{-1}$ mode is plotted versus temperature in Fig. 3 for stoichiometric, congruent, $\mathrm{ZnO}$ doped, and $\mathrm{MgO}$ doped $\mathrm{LiNbO}_{3}$. The solid line represents the width of the main line of stoichiometric $\mathrm{LiNbO}_{3}$ calculated from Eq. (1). At temperatures below $100 \mathrm{~K}$ there is good agreement between the calculated solid line and the experimental points in stoichiometric $\mathrm{LiNbO}_{3}$. With increasing temperature the side lines become important. This leads to a larger measured overall linewidth in Fig. 3 compared with the calculated linewidth of the single transition $v=0 \rightarrow 1$ (main line). When the Raman line shapes are calculated as described above, including the side lines, there is good agreement between the measured points in Fig. 3 and the calculated linewidths for stoichiometric $\mathrm{LiNbO}_{3}$.

The linewidths of the congruent and doped $\mathrm{LiNbO}_{3}$ crystals are much larger than that of the stoichiometric crystal (see Fig. 3). The dotted lines, which represent their temperature dependence, run nearly parallel to each other, corresponding to the addition of a temperature independent term whose magnitude is different for the various crystals. We attribute this term to the scattering of the optical phonons at crystal imperfections. The temperature independent part $\Gamma_{i}$ of the Raman linewidth of the $251 \mathrm{~cm}^{-1}$ mode was determined from Fig. 3 (at $20 \mathrm{~K}$ ) to be 5.5, 10.0, and $12.0 \mathrm{~cm}^{-1}$ for congruent $\mathrm{LiNbO}_{3}, \mathrm{ZnO}: \mathrm{LiNbO}_{3}$, and $\mathrm{MgO}: \mathrm{LiNbO}_{3}$, respectively. The corresponding numbers for the $273 \mathrm{~cm}^{-1}$ mode are $\Gamma_{i}=4.7,7.5$, and $7.5 \mathrm{~cm}^{-1}$. The results show that the density of imperfections increases in the sequence congruent, $\mathrm{ZnO}$ doped and $\mathrm{MgO}$ doped $\mathrm{LiNbO}_{3}$.

Using this information we have carried out a detailed analysis of the Raman line shapes of the 251 and $273 \mathrm{~cm}^{-1}$ modes in the congruent and doped crystals with the model of the main and side lines discussed above. We used the numbers for $\Gamma_{d}, \overline{\nu_{0}}, \Delta$, and $x_{e}$ obtained for the stoichiometric $\mathrm{LiNbO}_{3}$ crystal, i.e., the anharmonic decay of the optical phonons and the anharmonic potential of the side lines were taken to be the same for all crystals. The only difference for the stoichiometric crystal was that we added the temperature 

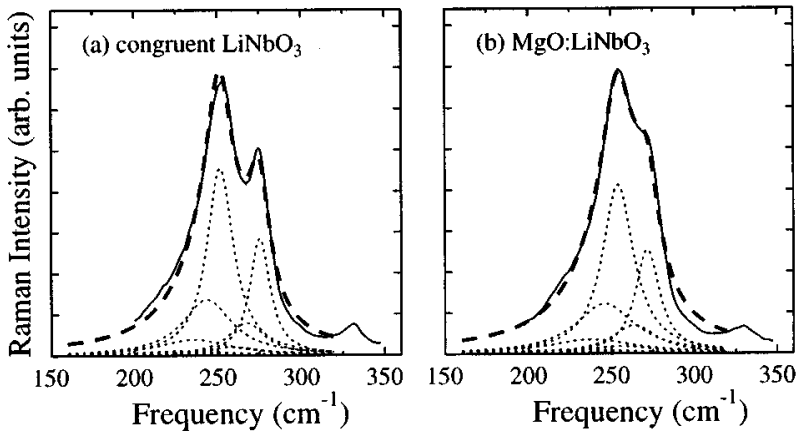

FIG. 4. Spontaneous Raman spectra of the $251 \mathrm{~cm}^{-1}$ and 273 $\mathrm{cm}^{-1}$ modes in congruent $\mathrm{LiNbO}_{3}$ and $\mathrm{MgO}: \mathrm{LiNbO}_{3}$ at $T=293 \mathrm{~K}$; linestyles as in Fig. 2.

independent term $\Gamma_{i}$ to the linewidth $\Gamma$ of the main line [in Eqs. (1) and (6)]. The results are shown for the 251 and 273 $\mathrm{cm}^{-1}$ modes of congruent $\mathrm{LiNbO}_{3}$ and $\mathrm{MgO}: \mathrm{LiNbO}_{3}$ at room temperature in Figs. 4(a) and 4(b). The dashed line is the sum of the dotted lines, which represent the main and side lines. There is good agreement between the solid experimental curve and the calculated dashed curve. A similar good agreement was found for the line shapes in all examined crystals from 20 to $300 \mathrm{~K}$, indicating that our model is a good representation not only for the stoichiometric but also for the congruent and doped crystals.

In summary, we have presented a model for the Raman line shapes in $\mathrm{LiNbO}_{3}$ which describes correctly the tem- perature dependence over a wide range for undoped and doped crystals. The Raman lines have been calculated by the superposition of closely spaced main and side lines corresponding to the transitions between the energy levels in an anharmonic potential for the ionic motion. The anharmonicity of the potential, which is important for models of ferroelectric properties of $\mathrm{LiNbO}_{3}$, was calculated from the experimental results. The widths of the main lines in stoichiometric $\mathrm{LiNbO}_{3}$ are determined by the decay of the optical phonon into two acoustic phonons. In congruent and doped $\mathrm{LiNbO}_{3}$ a temperature independent term was added to this linewidth, which was attributed to the scattering of the optical phonons at crystals imperfections.

We did not observe a frequency shift of the investigated phonon modes beyond that caused by the decay into two acoustic phonons. So we see no hint for a soft mode behavior. Comparison of the Raman spectra with the imaginary part of the dielectric susceptibility derived in model calculations for the displacive and order/disorder phase transition ${ }^{6}$ shows a better agreement with the latter. An examination of stoichiometric $\mathrm{LiNbO}_{3}$ at high temperatures and a comparison of the measured anharmonicity with theoretical calculations of the anharmonic potential ${ }^{7}$ will complement our understanding of the ferroelectric phase transition.

The authors gratefully acknowledge valuable help by Professor O. F. Schirmer and Dr. G. I. Malovichko, who lent us the stoichiometric crystals. We would also like to thank R. Pfister who participated in the experiments. This work was supported by the Deutsche Forschungsgemeinschaft.
${ }^{1}$ W. D. Johnston, Jr. and I. P. Kaminow, Phys. Rev. 168, 1045 (1968).

${ }^{2}$ Y. Okamoto, Ping-chu Wang, and J. F. Scott, Phys. Rev. B 32, 6787 (1985).

${ }^{3}$ Yu. K. Voron'ko, A. B. Kudryavtsev, V. V. Osiko, A. A. Sobol', and E. V. Sorokin, Sov. Phys. Solid State 29, 771 (1987).

${ }^{4}$ S. Kojima, Jpn. J. Appl. Phys. 32, 4373 (1993). Figures 3 and 4 in this work are interchanged.

${ }^{5}$ J. Mendes-Filho, V. Lemos, and F. Cerdeira, J. Raman Spectrosc. 15, 367 (1984).

${ }^{6}$ H. J. Bakker, Phys. Rev. B 52, 4093 (1995).

${ }^{7}$ I. Inbar and R. E. Cohen, Phys. Rev. B 53, 1193 (1996); R. E. Cohen, Nature 358, 136 (1992).

${ }^{8}$ The potential has also been interpreted in the framework of an extended mode representation (phonon picture) (Ref. 9).

${ }^{9}$ C. M. Foster, M. Grimsditch, Z. Li, and V. G. Karpov, Phys. Rev. Lett. 71, 1258 (1993); C. M. Foster et al., Phys. Rev. B 48, 10160 (1993).

${ }^{10}$ G. I. Malovichko et al., Appl. Phys. A 56, 103 (1993).

${ }^{11}$ U. T. Schwarz and Max Maier, Phys. Rev. B 53, 5074 (1996).

${ }^{12}$ C. Cohen-Tannoudji, B. Diu, and F. Laloë, Quantum Mechanics, 2nd ed. (Wiley, New York, 1977), p. 1110.

${ }^{13}$ G. W. Flynn, C. S. Parmenter, and A. M. Wodtke, J. Phys. Chem. 100, 12817 (1996).

${ }^{14}$ A. S. Pine and P. E. Tannenwald, Phys. Rev. 178, 1424 (1969).

${ }^{15}$ X. Yang, G. Lan, B. Li, and H. Wang, Phys. Status Solidi B 141, 287 (1987). 